\title{
Dynamics of Process Formation during Differentiation of Tectal Neurons in Embryonic Zebrafish
}

\author{
Rolf J. Kaethner, * Claudia A. O. Stuermer \\ Faculty of Biology, University of Konstanz, D-78434 Konstanz, Germany
}

Received 30 August 1996; accepted 7 January 1997

\begin{abstract}
Neurons acquire their distinct shapes after passing through many transitional stages in early development. To reveal the dynamics and spatiotemporal sequence of process formation in situ, the growth of neurons in the optic tectum of live zebrafish embryos ( 54 to $>100 \mathrm{~h}$ old) was monitored using time-lapse videorecordings. Neurons were labeled by injecting the fluorescent vital dye DiO into the cell-rich layer of the developing tectum in 50- to 70-h-old embryos. In phase 1, tectal neurons possess an apical "primary process" which reaches to the ventral aspect of the tectal neuropil. The primary process produces at its tip short transitory branches, some with growth cones, over a period of roughly $6 \mathrm{~h}$. One of the growth cones then elongates rapidly and grows toward the caudal tectum via a route characteristic of efferent axons. After retraction of excess branches and growth cones, branching activity resumes at the tip of the primary process to form the
\end{abstract}

dendritic tree (phase 2). The dendritic tree develops in the tectal neuropil through emission and retraction of many branches during a period of $>20 \mathrm{~h}$ (our longest continuous time-lapse movie). The tectal territory "explored" in this way is larger than the area finally covered by the tree resulting from growth and loss of branches. The dynamics observed here directly are probably characteristic for dendrite formation in vertebrates. Moreover, consistent with the sequence of neuronal differentiation observed in vitro, the growth of the axon precedes that of the dendrites, although both emerge from a common primary process in this type of tectal neuron. (c) 1997 John Wiley \& Sons, Inc. J Neurobiol 32: 627-639, 1997

Keywords: tectal neurons; in vivo development; zebrafish embryos; primary process; axogenesis; dendrite formation; transitory branches; time-lapse videorecordings

\section{INTRODUCTION}

The development of neurons and establishment of their complex, target-specific networks is a highly dynamic process during which each cell undergoes many changes in shape and size.

The typical events at the soma of differentiating

* Present address: Neurologische Universitätsklinik, University of Tübingen, Hoppe-Seyler-Str. 3, 72076 Tübingen, Germany

Correspondence to: C. A. O. Stuermer

Contract grant sponsor: Deutsche Forschungsgemeischaft; contract grant number: SFB 156

(C) 1997 John Wiley \& Sons, Inc. CCC 0022-3034/97/060627-13 neurons were analyzed in detail in hippocampal neurons in culture (Dotti et al., 1988) and confirmed in hypothalamic neurons (Diaz et al., 1992) and macroneurons of the cerebellum (Caceres et al., 1991). In these neurons, one of several minor processes differentiates into the axon, and this event is coupled to the acquisition of cell polarity (reviewed in Craig and Banker, 1994). Thereafter, the remaining minor processes differentiate into dendrites.

That axon outgrowth precedes the development of dendrites was also concluded from an analysis of fixed tissue at increasingly advanced stages of nervous system differentiation. A study of neurons in sections of such samples also indicated that both 
axonal and dendritic arborizations undergo substantial modification during development (reviewed in Berry, 1991), but the true dynamics of these events cannot be adequately represented by static images.

More recently, the growth of fluorescently labeled axons in embryos and the formation of their axon terminal arborizations was monitored directly using time-lapse videomicroscopy (Harris et al., 1987; O'Rourke and Fraser, 1990; Kaethner and Stuermer, 1992, 1994a; O'Rourke et al., 1994), and emission and retraction of branches are recognized as normal exploratory events during formation of retinal axon arborizations in the optic tectum of fish and frogs. In fish, many of the tectal neurons contacted by retinal axons have their somata in the lowest of the tectal layers, i.e., the stratum periventriculare (SPV), and their apical dendrites in the upper layers of tectum (Vanegas et al., 1974; Meek, 1983). Most of these neurons in the SPV have efferent axons to various targets (Meek, 1983; Davis and Northcutt, 1983). These axons derive from the dendrite (Meek, 1983; Meek and Schellart, 1978), whereas the classical textbook neuron produces its axon from the pole opposite the dendrite (reviewed in Berry, 1991). The former is a typical neuronal form in the optic tecta of frogs (Hughes, 1990) and birds (Cuénod and Streit, 1979) and also occurs in the mammalian hippocampus (Ramon y Cajal, 1911). Whether the sequence of axogenesis and dendritic tree development of such neurons obeys similar developmental rules as the "model" neuron, in which the axon forms first and the dendrite is produced later, is unknown.

In this study, we monitored the development of tectal neurons in zebrafish between 54 and $>100 \mathrm{~h}$ postfertilization. In the developing zebrafish optic tectum, most of the neuronal somata are clustered in a cup-shaped array with the "neuropil" (Stuermer, 1988 ) at its center, where retinal axon arbors are forming. Through injection of a $\mathrm{DiO}$ solution into the cell-rich layer of the tectum, we were able to label individual neurons and record their developmental growth activities with time-lapse videomicroscopy in living zebrafish.

When growing into the tectal neuropil, the dendrites of these neurons extend and retract branches in a manner resembling that of retinal axon arbors. Prior to dendrogenesis, these neurons extend their efferent axons from the primary process which subsequently gives rise to the dendrite. However, the emergence of the axon is also preceeded by phases of exploratory branching of the primary process. Thus, the present recordings reveal the emergence and growth of the efferent axon of tectal neurons and demonstrate the impressive branching activity of their dendritic trees in the tectal neuropil.

Part of these data were previously presented as an abstract [Kaethner and Stuermer (1994b) Soc. Neurosci. Abstr. 20: 1081].

\section{METHODS}

Zebrafish embryos were obtained from our breeding colony and staged. Maintenance of adult fish and embryos was as described earlier (Kaethner and Stuermer, 1992). Observations of living dendrites were performed in the zebrafish mutant strain golden which has - in contrast to wild-type embryos - transparent melanophores. Since the golden melanophores bear fewer black pigment granules, labeled tectal cells can easily be seen through the unopened skull. For experimental manipulations prior to hatching, which occurs from about $72 \mathrm{~h}$ postfertilization ( $\mathrm{hpF}$ ) onward, embryos were removed from their egg cases with pointed forceps and kept in water in small petri dishes.

\section{Dye Injections}

Embryos were anesthesized in $0.03 \%$ methansulfonate, 3-aminobenzoic acid ethylester (MS 222; Sigma) for the injection procedure and during time-lapse recordings. They were transferred to a coverslip in a position that allowed a dorsal view of the tectal hemisphere, which received the dye. Most of the surrounding water was removed, except for a drop around the embryo. A silicon ring was placed around the embryo to prevent it from drifting and filled with oxygen-diffusible voltalef oil (Atochem), allowing normal progression of the embryo's development (Kaethner and Stuermer, 1992). In each experiment, injections were made in 10 embryos of one batch, of which only one was selected for videorecordings.

Next, $0.5-30 \mathrm{pL}$ of a saturated solution of the vital dye $\mathrm{DiO}$ (di-octadecylindocarbocyanine toluene sulfonate; Serva) in cold liver oil (Sigma) were pressure injected with a picospritzer into the cell-rich layer of the dorsal optic tectum (Fig. 1). This resulted in the labeling of one or more cells. Here, videorecordings and further analysis are discussed of only those embryos in which individual neurons/cells were discernible. However, recordings were performed on 76 successfully labeled neurons. Those not included in this analysis were embryos with groups of two, three, or more labled neurons whose processes could not be assigned to an individual cell and embryos in which neurons were videorecorded over short time periods $(<5 \mathrm{~h})$. Videorecordings of joined cellular processes as well as those of shorter duration all showed qualitatively the same behavior of cellular process formation 

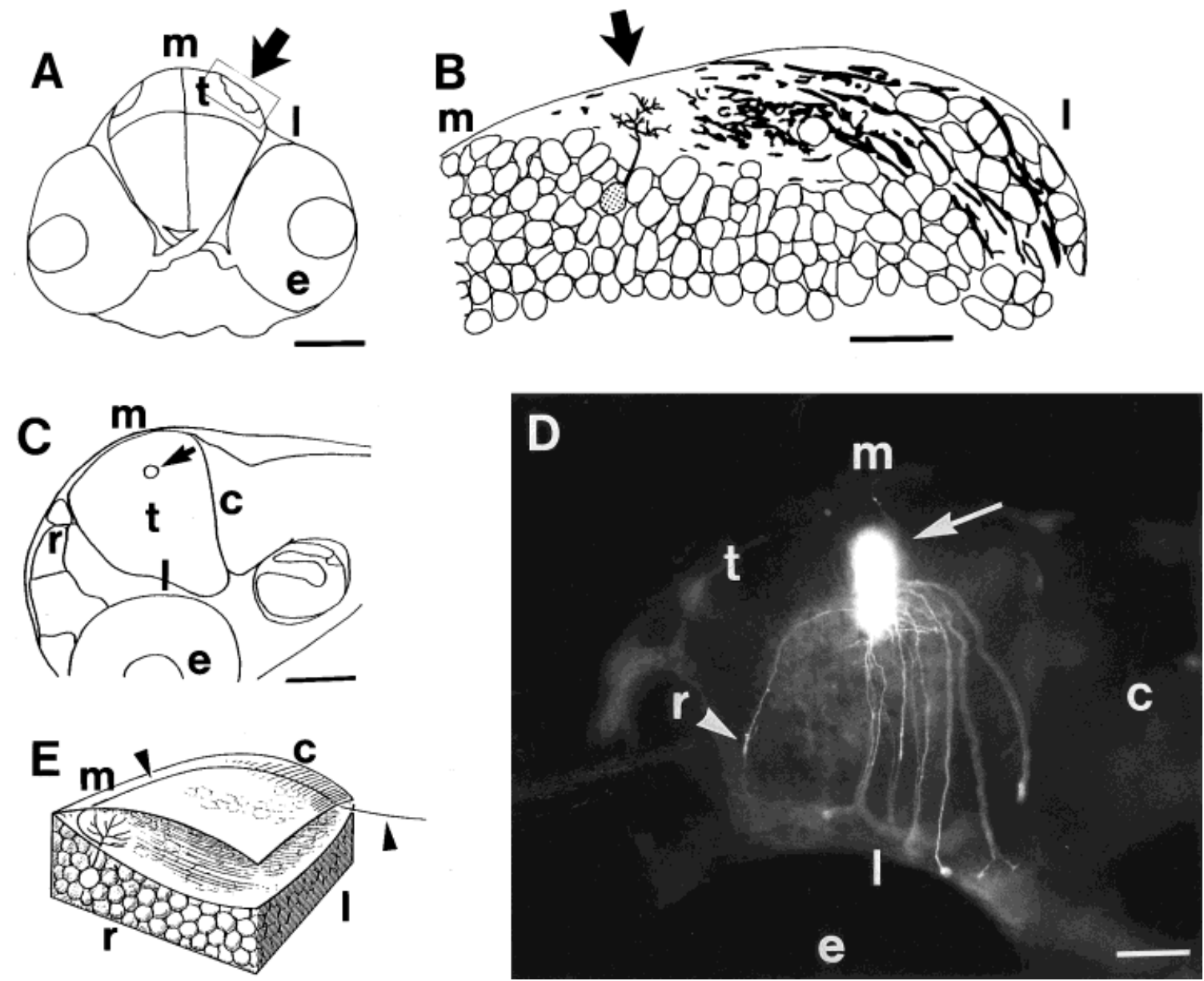

Figure 1 Location of labeled tectal neurons and their efferent axons. (A) Cross section through the head of a zebrafish embryo showing the location of the tectum $(\mathrm{t})$ and its neuropil. The arrow indicates the lens and the angle of view into the tectum. Scale bar-100 $\mu \mathrm{m}$. The boxed area is enlarged in (B). (B) A more detailed drawing of the area of the tectum framed in $(\mathrm{A})$. The roundish features represent the neuronal somata. The shaded soma show the location of a typical labeled neuron and the idealized shape of its dendrite in the tectal neuropil. The retinal axons entering the neuropil are represented as thick black lines. The spatial organization of neuronal somata and retinal axons was drawn from a section with axons labeled by horseradish peroxidase. The large arrow represents the lens and angle of view as in (A). Scale bar-20 $\mu \mathrm{m}$. (C) Lateral view of the zebrafish head. The site of dye injection is marked by an arrow. Scale bar-100 $\mu \mathrm{m}$. (D) Lateral view, same as in (C), showing the deposition of a large amount of dye (arrow) in the dorsomedial tectum, and the routes of the labeled efferent axons into the caudal, rostral (arrowhead), and toward the ventrolateral tectum. Scale bar-50 $\mu \mathrm{m}$. (E) Three-dimensional sketch of the cell-rich layer of the tectum, the neuropil, a labeled neuron (white cell body) with its dendrite in the neuropil, and the efferent axon (arrowhead) coursing into caudal tectum. e-eye; t-tectum; m-dorsomedial; l-ventrolateral; r-rostral; c-caudal.

as detailed here in individual cells. Injections were done in embryos between 50 and $70 \mathrm{hpF}$. In table 1, fish are identified by their age $(\mathrm{hpF})$ at the beginning of the recordings. Additional labeled cells and their processes were viewed and analyzed in embryos between 50 and $110 \mathrm{hpF}$, which were labeled at the same time as their age-matched counterparts used for continuous time-lapse recordings. The mor- phology of the labeled neurons in these embryos was viewed at two different time points correlating with the beginning and end of the videorecordings in the selected embryos. Size of cells and their morphology, including length and number of branches, point of origin of the axon, and axonal pathway, were all as seen in the age-matched embryos subjected to the videorecordings. 
Table 1 Individual Neurons and Their Dendritic Trees

\begin{tabular}{|c|c|c|c|c|c|c|}
\hline \multirow[b]{2}{*}{ Neuron } & \multirow{2}{*}{$\begin{array}{c}\text { Observation } \\
\text { Period (h:min) }\end{array}$} & \multirow{2}{*}{$\begin{array}{c}\text { Age of } \\
\text { Fish (hpF) }\end{array}$} & \multicolumn{2}{|c|}{ Size of Exploration Fields } & \multicolumn{2}{|c|}{ Size of Dendrites } \\
\hline & & & (\% Tectum) & $\mathrm{la} \times \mathrm{sa}(\mu \mathrm{m})$ & (\% Tectum) & $\mathrm{la} \times \mathrm{sa}(\mu \mathrm{m})$ \\
\hline 1 & $8: 12$ & 65 & & & & \\
\hline 2 & $8: 11$ & 58 & & & & \\
\hline 3 & $16: 12$ & 74 & & & & \\
\hline 4 & $11: 48$ & 76 & & & & \\
\hline 5 & $10: 24$ & 99 & & & & \\
\hline 6 & $24: 05$ & 54 & 0.5 & $20.0 \times 15.0$ & 0.2 & $18.7 \times 13.7$ \\
\hline 7 & $10: 10$ & 81 & 1.6 & $24.0 \times 26.0$ & 1.0 & $12.0 \times 23.0$ \\
\hline 8 & $18: 51$ & 80 & 1.5 & $21.3 \times 31.3$ & 1.0 & $22.0 \times 43.0$ \\
\hline 9 & 16:08 & 70 & 1.0 & $22.7 \times 23.7$ & 0.8 & $25.7 \times 21.7$ \\
\hline 10 & $19: 10$ & 74 & 0.8 & $24.3 \times 22.0$ & 0.4 & $17.0 \times 18.7$ \\
\hline 11 & $16: 00$ & 75 & 2.2 & $29.3 \times 41.7$ & 1.2 & $34.7 \times 39.3$ \\
\hline 12 & $16: 42$ & 75 & 0.6 & $18.0 \times 16.3$ & 0.3 & $13.3 \times 11.0$ \\
\hline 13 & $5: 00$ & 77 & 1.0 & $21.7 \times 20.3$ & 0.9 & $15.7 \times 27.3$ \\
\hline 14 & $18: 51$ & 80 & 0.8 & $17.0 \times 24.7$ & 0.3 & $10.3 \times 21.3$ \\
\hline 15 & $18: 51$ & 80 & 1.4 & $20.3 \times 31.0$ & 1.0 & $35.3 \times 26.0$ \\
\hline 16 & $5: 33$ & 59 & 1.6 & $32.0 \times 23.7$ & $*$ & $*$ \\
\hline 17 & $20: 46$ & 62 & 1.3 & $22.3 \times 31.3$ & $*$ & $*$ \\
\hline $18^{*}$ & $6: 40$ & 78 & 1.4 & $26.0 \times 25.0$ & $*$ & $*$ \\
\hline $19 *$ & $6: 40$ & 78 & 1.4 & $26.0 \times 25.0$ & $*$ & $*$ \\
\hline $20 *$ & $6: 40$ & 78 & 1.4 & $26.0 \times 25.0$ & $*$ & $*$ \\
\hline 21 & & 77 & & & 0.4 & $14.7 \times 8.7$ \\
\hline 22 & & 77 & & & 0.5 & $8.3 \times 37.0$ \\
\hline 23 & & 80 & & & 0.4 & $15.7 \times 27.3$ \\
\hline 24 & & 80 & & & 0.4 & $11.7 \times 16.7$ \\
\hline 25 & & 80 & & & 0.2 & $11.7 \times 6.3$ \\
\hline
\end{tabular}

Data show identification of individual neurons (column 1), length of continuous time lapse recordings in hours and minutes (column 2), and the age of the zebrafish embryo at the beginning of the recordings (in hpF) (column 3). Columns 4 and 5 give the dimensions of the dendritic exploration field, expressed as tectal neuropil area $(2,3)$, and as the length of its short axis (sa) and long axis (la). The developing dendrites of neurons 18-20 (marked by an asterisk in column 1) interdigitated so that the exploration field of these neurons is given as a value of all three together. In columns 6 and 7, the sizes of the dendrites at the end of the time-lapse recordings are listed as percent tectal neuropil area and by the length of their short (sa) and long axes (la). Dendrites of neurons 16-20 (asterisk in columns 6 and 7) were not measured. As was the case for neurons 18-20, processes of neurons 16 and 17 partially interdigitated and could no longer be distinguished when recording was terminated. In neurons $1-5$, branching activity of the primary process, but not that of the dendritic tree, was recorded.

\section{In Vivo Time-Lapse Video Microscopy}

The coverslip with an embryo which had received a dye injection was mounted on a slide and placed onto the stage of the microscope. All embryos were positioned so that the lens of the microscope was at right angle to the surface of the tectum, as indicated in Figure 1. Thus, labeled neurons were viewed directly from above (Fig. 1) like a bird's-eye view of a growing tree. Cells which had incorporated the dye into their membranes were viewed through the $\times 20, \times 40$, and $\times 100$ oil-immersion lenses of a fluorescence microscope (Zeiss Axioplan). Recordings were performed with the $\times 100$ lens. The microscope was equipped with the appropriate filters for fluorescence, an infrared filter, a 100-W Xenon lamp as the light source, and a silicone intensified target (SIT) camera. To avoid photodamage of the neurons, the light intensity was low. A shutter, which opened every $30 \mathrm{~s}$ for $200 \mathrm{~ms}$, was inserted into the light path. Three frames were shot within the $200 \mathrm{~ms}$, digitally averaged and enhanced (Kaethner and Stuermer, 1992), and recorded by a video recorder (U-matic). Recordings were carried out as long as the blood circulation was normal (Kaethner and Stuermer, 1992, 1994). Time-lapse recordings were terminated when the blood flow ceased and no further measurements were made in these cases (neurons 16 and 17 of Table 1). In two embryos (77 and $80 \mathrm{hpF}$; Table 1 ), only the extent of dendrites (of neurons 21-25) was determined (not their development), because the focus was on another cell/dendrite (i.e., nos. 13-15, respectively).

Moreover, some neurons in living fish may abruptly cease to grow, probably owing to photodamage. In these instances, recording was terminated immediately. Neu- 
ronal processes which exhibited a beaded appearance at the end of the recording session were not used for further evaluations. Recording was also terminated if the appearance of other labeled neuronal processes hampered the view of the neuron under consideration. This occurred at the end of the recordings of neurons 16-20 (Table 1), whose dendritic tree sizes could not be determined because processes of other labeled neurons had interdigitated with those of the neurons of interest.

As in our earlier studies (Kaethner and Stuermer, 1992, 1994a), we used DiO rather than DiI to label the neurons. It was observed that DiI creates photoradicals more quickly than DiO, leading to a more rapid disintegration of the neuron under observation (Kaethner and Stuermer, 1992). That the dye did not interfere with the neuron's normal development (except for instances mentioned above) is deduced from their branching activity and growth cone formation, which cease when the cell dies. Moreover, in pioneering experiments for this study, longer intervals (60 s and $2 \mathrm{~min}$ ) between the 200-ms illumination periods were used, and as in the study on retinal axon growth (for detailed discussion, see Kaethner and Stuermer, 1992) no differences were noted in morphology or dynamic turnover of branches of labeled cells. Furthermore, the morphology of cells under continuous observation was similar to the morphology of cells in age-matched embryos not used for long videorecordings (see above). This, together with results of earlier reports (Harris et al., 1987; O'Rourke and Fraser, 1990; Kaethner and Stuermer, 1992), indicates that it is unlikely that the content of fluorescent dyes affects the normal behavior of neurons (Honig and Hume, 1986). Recordings with shorter intervals were preferred because, as demonstrated in Figure 4, for instance, new branches arise or are retracted within $30 \mathrm{~s}$.

Boundaries of the tectal neuropil and adjacent brain areas are visible through the $\times 20$ lens with Nomarski optics, allowing determination of the tectal position of the recorded neurons and their processes. To illustrate the morphology of the recorded cells (viewed from above), the entire extent of the dendritic tree and the labeled neuron was registered in different focal planes by focusing through the neuropil and cell layer stepwise at the beginning and end of the recording session. In drawings, dendritic trees are represented in a planar projection and neurons and dendrites shown in an upright position.

The sizes of dendritic trees and their exploration fields were assessed by two methods (Kaethner and Stuermer, 1992, 1994a). Lines were drawn to connect the tips of the branches or fields, and the short and long axes of the resulting shapes (Stuermer, 1984, 1988) were measured. The long axis was defined by the orientation of the primary process and the short axis was perpendicular to it. To approximate the area, grids of equal-sized squares were placed over the drawings of dendritic trees and exploration fields. All squares occupied by branches were counted and added, and the sizes calculated. All areas are expressed as a percentage of the neuropil area which was determined in the same way (Kaethner and Stuermer, 1992, 1994a).

\section{RESULTS}

The organization of the embryonic zebrafish optic tectum, the sites of dye injections, the location and form of $\mathrm{DiO}$ labeled neurons, and the path of their efferent axons are illustrated in Figure 1. The retinal axons were labeled by horseradish peroxidase (HRP) application to the retina (Stuermer, 1988) to visualize the location and extent of the tectal neuropil, and to indicate the relationship between retinal axons and dendrites of tectal neurons in the neuropil [Fig. 1(B)]. Neurons labeled by DiO injection into the dorsal tectum [Fig. 1(B,C,E)] project dendrites radially toward the surface of the brain and are observed directly from above. Their axons project either in a curved path along the dorsomedial rim to the caudal end of the tectum or straight to the ventrolateral tectal border. Less frequently, they project into the rostral tectum [Fig. 1(D)]. When viewed from above, the focus was kept on the most active portions of the developing processes. Monitoring events of interest with the $\times 100$ oil-immersion lens allows observation of a significant portion, but sometimes not all, of the dendrite's extent. Moreover, when recording the dendritic branching activity in the neuropil, events occurring at and near the soma and primary process are missed. Still, typical aspects of neuron development could be determined by recording several neurons at different stages of their growth. Many neurons were recorded over short periods. They, as well as labeled neurons in small groups, are omitted from the quantitative evaluation of process formation and from Table 1, although events observed in their processes were qualitatively similar to those of neurons observed over longer periods. We also recorded cells with small motile branches more or less evenly distributed over their radial process. These are likely to be radial glial cells and are not considered further.

Time-lapse recordings of 20 individually labeled neurons (identified in Table 1 ) of 16 zebrafish (selected from more than 76 labeled neurons ) revealed two significant phases of early development. Phase 1 is characterized by the extension of a primary process in the direction of the neuropil, exploratory branching at its tip, and the departure of the efferent 


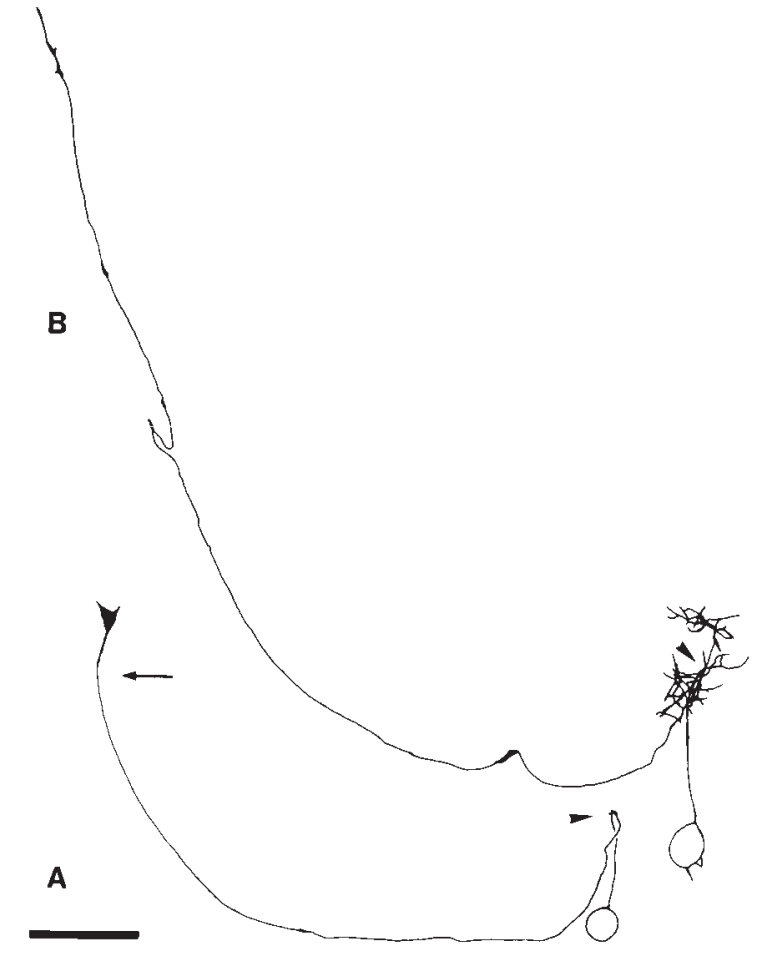

Figure 2 Primary process, origin of the axon and dendritic tree. (A) Morphology of neuron 6 after the axon (arrow) has migrated away from its cell of origin. The growth cone originated from the tip of the primary process (arrowhead) and was monitored until it reached the length shown in (B) New branches emerging from the tip of the primary process were videorecorded for over 8 h. the beginning, the neuron exhibited the shape illustrated in (A). Scale bar-20 $\mu \mathrm{m}$.

axon from it (Fig.2; also see also Fig. 7). Phase 2 consists of the emergence and dynamic modelling of the dendritic tree in the tectal neuropil (see Fig. 7).

Recordings of most neurons in this study began after they had extended a process toward the neuropil. This first extension is called the primary process. For one neuron (no. 4, Table 1), recording began prior to primary process formation. This neuron had small branches around its circumference but no significant primary process. It resembled a developing hippocampal neuron in culture at stage 1 (Dotti et al., 1988) and suggests that transient events in the soma may precede process formation in vivo as well as in vitro (Dotti et al., 1988; Deitsch and Banker, 1993).

When the primary process reaches the ventral border of the tectal neuropil, it begins to extend and retract branches and growth cones. These exploratory branching events were recorded continuously in the case of neurons 1-6 (Table 1), focusing on the primary process, and is shown in Figure 3. The exploratory events, which last roughly $6 \mathrm{~h}$, finally result in the rapid extension of a single growth cone. This rapid extension was recorded in neuron 6 (Fig. 2), neuron 1 (Fig. 3), and neuron 5. The growth cones grew toward the caudal border of the tectum in a curved path [Fig. 1(D,E)] with an average velocity of $30 \mu \mathrm{m} / \mathrm{h}$ without extending side branches en route. Axons of neurons 2, 3, and 4 were seen departing from the primary process at the end of the videorecording. These axons grew rather directly to the ventrolateral tectum [Fig. 1(D)]. Similar events at the primary process were observed in 15 other neurons, and images of these confirmed the presence of branches and growth cones, the point of origin of the axon, and the axonal pathways.

Upon growth cone elongation toward the caudal tectum, the earlier branches and growth cones at the tip of the primary process retracted as shown in neuron 1 (Fig. 3). These events were also recorded in neurons 2, 3, and 4. Accordingly, the tip of the primary process of neuron 1,5 , and 6 (Fig. 2) was free of branches (except for a few thorns), at the time at which the growth cone of the rapidly elongating axon had moved away from its point of origin. Retraction of exploratory branches and growth cones at the tip of the primary process marks the end of phase 1 , which lasts approximately $15 \mathrm{~h}$. The events characteristic of phase 1 resemble to some extent those observed with hippocampal neurons in culture at stage 3 (Dotti et al., 1988), where one of the neurites of the cell differentiates into a rapidly elongating axon. The remaining processes of hippocampal neurons develop into dendrites at stage 4 (Dotti et al., 1988). The tectal neurons, however, differ from hippocampal neurons in culture in that the axon departs from the single primary process rather than from the soma, and the remaining branches are retracted before new ones emerge to form the dendrites. Thus, a transient phase of local exploration appears to be terminated by the rapid extension of one of the growth cones and the retraction of other growth cones and branches at the tip of the primary process (see Fig. 7).

Branching activity then resumes at the tip of the primary process. This and the subsequent events of phase 2 lead to the elaboration of the dendritic tree as was observed in neurons 1-6. Branching of neuron 6 continued over several hours, resulting in the dendritic tree morphology represented in Figure 2 at the end of the (24-h) recording.

The elaboration of the dendritic tree is character- 

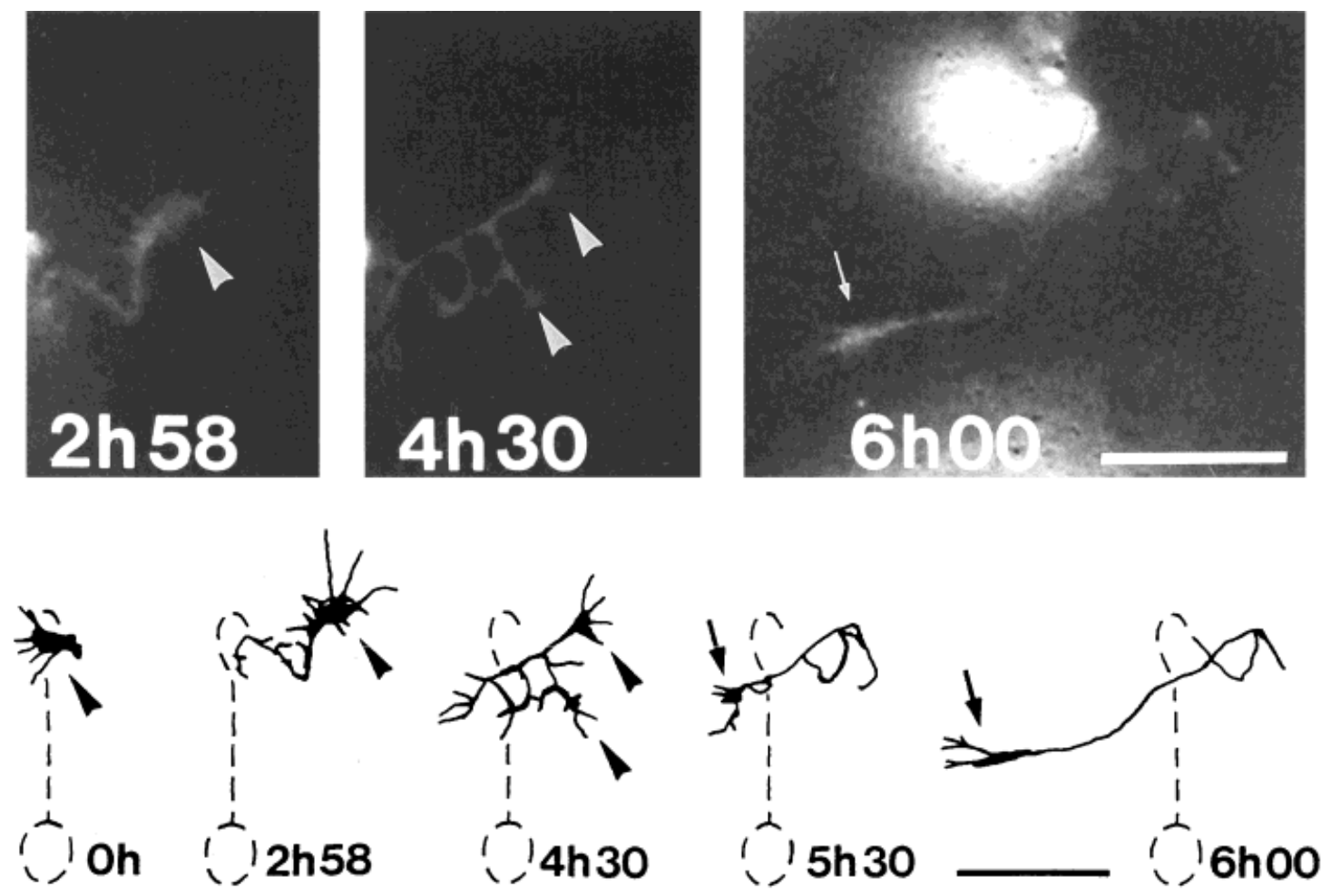

Figure 3 Exploratory branching and axogenesis. Branches and growth cones emerging at the tip of the primary process of neuron 1 were recorded continuously for more than $6 \mathrm{~h}$ (Table 1 ) and are documented in drawings and corresponding video images. Times are given in hours and minutes. Several growth cones (arrowheads) and branches appear before the growth cone (arrow) elongates and grows away from its site of origin. Cell body and primary process are indicated by dotted lines. Scale bar-20 $\mu \mathrm{m}$.

ized by the repeated emission and retraction of numerous side branches. In contrast to branches in phase 1 , none of those appearing in phase $2 \mathrm{had}$ growth cones, as seen in case of neurons 6-20 and in shorter sequences of additional neurons.

Neurons 7-20 already had small dendritic trees in the tectal neuropil when they were first identified and recorded. Since the branching activity occurred in the portion of the dendrite occupying the neuropil, the focus was kept on this portion of the dendrite during further recording. All neurons exhibited characteristic branching activity involving emission and retraction of branches.

The length changes of individual branches occurred within minutes, as is documented by selected images from a continuous movie of $10 \mathrm{~h}$ in Figure 4. Selected images of a continuous recording of neuron 7 demonstrate the larger-scale changes of the dendritic tree over a 5 -h period (Fig. 5). Branches present earlier [Fig. 5(A,B) and $5\left(\mathrm{G}, \mathrm{G}^{\prime}\right)$ ] have disappeared $30 \mathrm{~min}$ to $1 \mathrm{~h}$ later [Fig. 5(C,E,D) and 5(H, $\left.\mathrm{H}^{\prime}, \mathrm{H}^{\prime \prime}\right)$. New

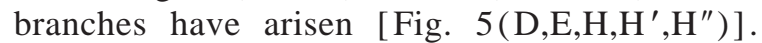
Thus, the dendritic tree rapidly and continuously changes its morphology through production and loss of branches. The dendrite of neuron 7 produced 14.2 new branches/hour and retracted 12.5. The average determined from 12 neurons (no. 617; Table 1) is $10.2( \pm 3.7)$ new branches/hour. The average of branches retracted per hour was $9.0( \pm 3.3)$. During these events, the dendrites increase in length and undergo dramatic changes in shape (Figs. 5-7 and Table 1). This resembles retinal axon terminal arbors which form through extension and retraction of branches in the tectal neuropil (Kaethner and Stuermer, 1992, 1994a). Both go through a phase of exploratory growth. The exploration field determined earlier for retinal axon arbors is the area of tectal neuropil which is touched by branches, transient or stable, during 

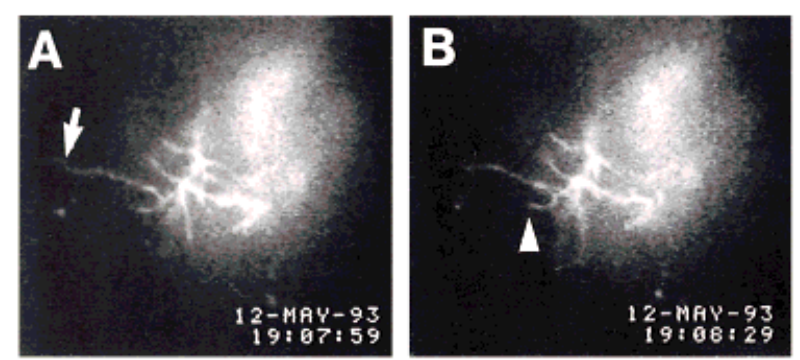

A'

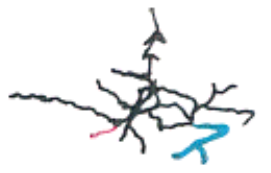

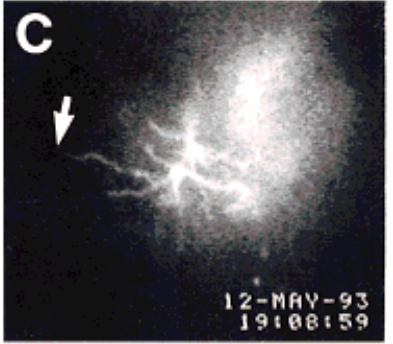

C'

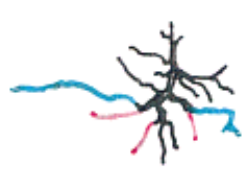

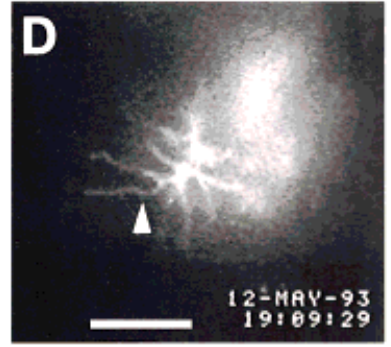

D'

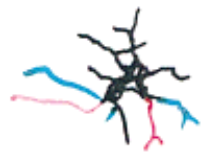

Figure 4
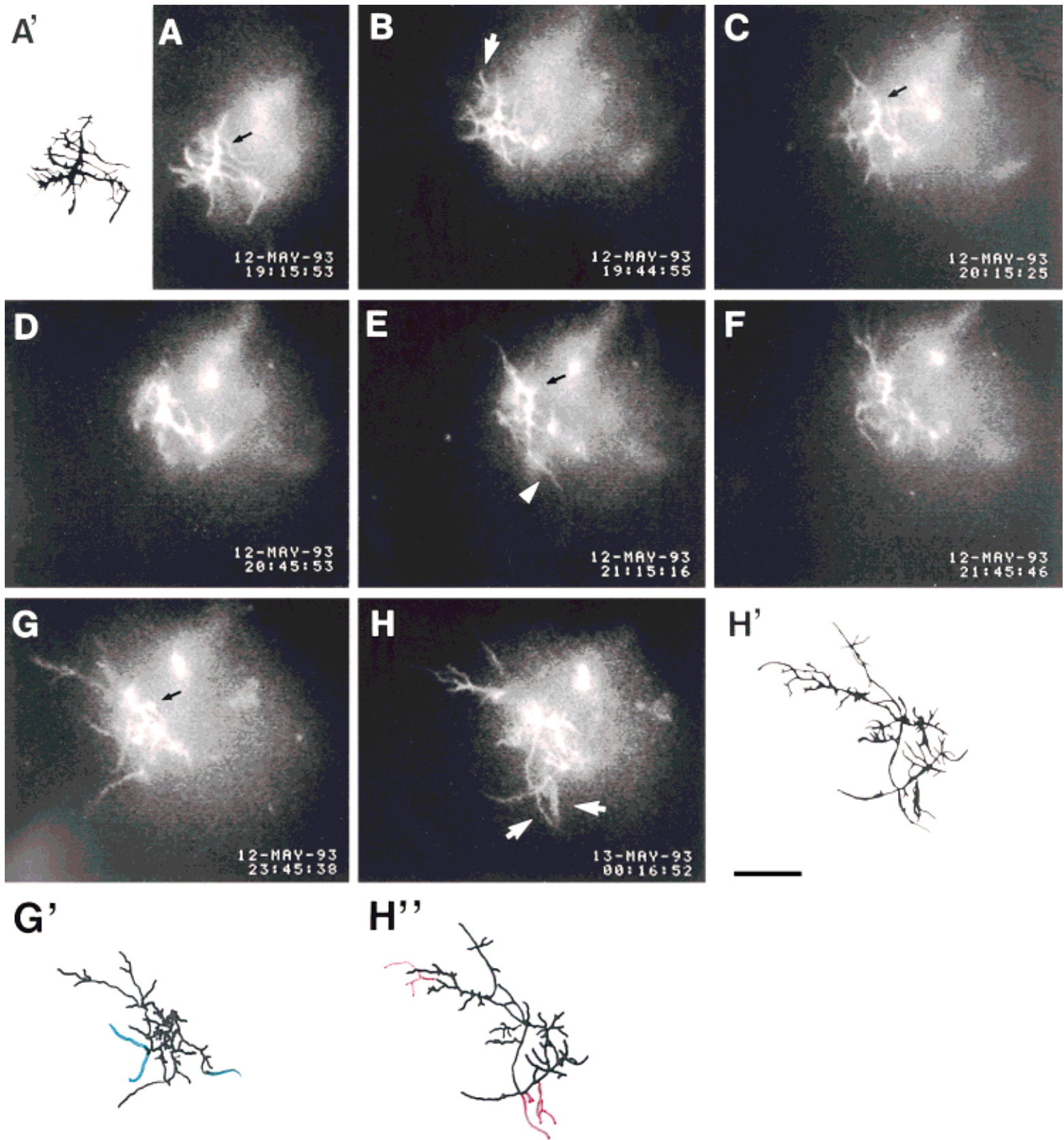
the time of observation (Kaethner and Stuermer, 1992, 1994a).

The exploration fields of the dendritic tree were determined as previously described for the axon arbors (Kaethner and Stuermer, 1992). All branches observed in the tectal neuropil during recording were drawn and represented in a planar projection. The areal extent was determined by two methods described earlier (Kaethner and Stuermer, 1992) (see Methods). Only that portion of the dendritic tree which was under continuous observation was included in these measurements, so the actual size of the dendritic exploration field may sometimes be underestimated. Also, the long and short axes of the dendrites determined at the end of the recordings can in certain instances be longer than the axes of the exploration fields, i.e., when the longest branches of large dendrites extend beyond the field of view chosen for filming (Table 1, neurons 11 and 15).

The partial exploration fields of the dendritic trees (Table 1) comprises between $0.5 \%$ and $2.2 \%$ of the tectal neuropil area. The area increase per hour averages $28.5 \%( \pm 11.5 \%)$ of the neuropil area that is covered by the tree at the end of the recording $(n=12)$. The latter was determined from drawings of the dendritic tree and its neuron of origin. The entire extent of the dendritic tree at the end of each recording covers between $0.2 \%$ and $1.2 \%$ of the neuropil area, and is thus smaller than its exploration field (Table 1).
A significant proportion of the branches visible at the end of the recording session was contained in the dendritic segment which was recorded. Events taking place outside the area seen by the lens and at the cell body and the proximal portion of the dendritic shaft (the former primary process) are missed. Nevertheless, the recordings cover a substantial proportion of the branching activity within the neuropil. When the labeled neurons were viewed at the end of the recordings, the portion of the dendrite nearest the soma (the former primary process) was free of branches in all cases examined (Figs. 6 and 7). Also, the efferent axon still originated from the dendrite, indicating that no dramatic changes occurred in the proximal portion of the dendrite while the dynamic changes in the distal portion were being recorded.

This, and the branching activity at the tip of the primary process prior to axogenesis, provide novel and direct documentation of the dynamics of early neuronal differentiation in the vertebrate central nervous system (CNS). The morphologic changes of tectal neurons in phase 1 and 2 are summarized in Figure 7.

\section{DISCUSSION}

Our time-lapse videorecordings have provided new insights into the sequence and dynamics of axogen-

Figure 4 Elongation and retraction of branches. The elongation of dendritic branches and their retraction occurs within minutes, as illustrated by selected images of a time-lapse recording of the dendritic tree of neuron 4 . The branch marked by arrows in (A) and (C) retracts during the $60 \mathrm{~s}$ between the two images. In $\left(\mathrm{B}^{\prime}, \mathrm{C}^{\prime}, \mathrm{D}^{\prime}\right)$, this retracting branch, as well as other retracting branches, is shown in green. The branch marked by an arrowhead in (B) and (D) elongates during the intermittent $60 \mathrm{~s}$ and is shown in red in $\left(\mathrm{A}^{\prime}, \mathrm{B}^{\prime}, \mathrm{C}^{\prime}, \mathrm{C}^{\prime}\right)$. Selected examples of other newly forming branches are also red. The bright area to the right of the dendrites results from the labeled cell body, other branches which are out of focus, and the dye deposit in the cell-rich layer deep to the dendrite. The date and time of the recordings appear at the lower right. Scale bar-10 $\mu \mathrm{m}$.

Figure 5 Changes in dendritic tree morphology. Emission and retraction of side branches during a 5-h period are documented by selected images from a 10-h continuous time-lapse movie of neuron 7. The dendritic tree of images $(A)$ and $(\mathrm{H})$ are also illustrated in drawings $\left(\mathrm{A}^{\prime}, \mathrm{H}^{\prime}\right)$. Examples of newly arising branches which were not present in the previous image are marked by white arrows $(\mathrm{B}, \mathrm{H})$ and those in $\left(\mathrm{H}, \mathrm{H}^{\prime}\right)$ not present in $\left(\mathrm{G}, \mathrm{G}^{\prime}\right)$ are shown in red in $\left(\mathrm{H}^{\prime \prime}\right)$. An example of branches which were subsequently retracted is marked by a white arrowhead $(\mathrm{E})$. Other examples of branches present in $\left(\mathrm{G}, \mathrm{G}^{\prime}\right)$ but absent in $\left(\mathrm{H}, \mathrm{H}^{\prime}, \mathrm{H}^{\prime \prime}\right)$ are shown in green in $\left(\mathrm{H}^{\prime \prime}\right)$. The black arrow $(\mathrm{A}, \mathrm{C}, \mathrm{E}, \mathrm{G})$ indicates branch points persisting throughout the period covered here. The areal extent of the dendritic tree in the tectal neuropil has increased $\left[\mathrm{cf} .\left(\mathrm{A}^{\prime}, \mathrm{H}^{\prime}\right)\right]$. The bright area in images $(\mathrm{A}-\mathrm{H})$ is from the cell body and the dye deposit at the injection site. The brightest dot is a mobile cell that has phagocytized the dye. Scale bar-10 $\mu \mathrm{m}$. 


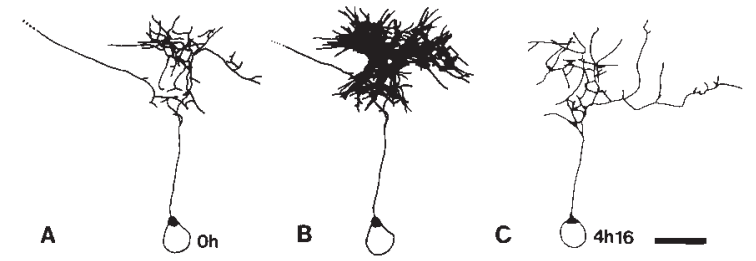

Figure 6 Dendritic tree and exploration field. Changes in shape of the dendritic tree of neuron 8 occurs over a period of $4 \mathrm{~h} 16 \mathrm{~min}(\mathrm{~A}, \mathrm{C})$ through growth and loss of branches. All branches in the segment under continuous observation, whether transient or stable, are represented in (B) and define the (partial) exploration field of this dendrite. The area of tectal neuropil covered by the final tree is two-thirds of the area of the partial exploration field, representing $1.5 \%$ of neuropil area. The long process to the left in (A) and (B) is the efferent axon. Scale bar-10 $\mu \mathrm{m}$.

esis and dendrite formation in tectal efferent neurons in their intact environment. Aside from short transitory branches at the soma, the first prominent extension of the tectal neurons is the single apical primary process. Only one of the many "exploratory" branches and growth cones that this primary process generates at its tip elongates to become the efferent axon. This very primary process also initiates dendritic branches, but only after axon outgrowth has occurred and after the earlier exploratory branches were retracted. The videorecordings also revealed characteristic events during formation of the dendritic tree which involves extension, modification, and retraction of numerous branches over several hours. We infer that this branching activity within the tectal neuropil is an exploration of the tectal environment.

The development of these tectal neurons resembles the development in culture of the most closely analyzed neuron types, the hippocampal neurons and cerebellar macroneurons (reviewed in Craig and Banker, 1994). In both, the axon differentiates prior to the dendrites. The differentiation of the axon in both types of neuron is concomitant with acquisition of neuronal polarity. This was demonstrated by the appearance of Gap-43 in the axon (Goslin et al., 1988), by the specific organization of microtubules (Burton and Paige, 1981; Heidemann et al., 1981; Baas et al., 1989), and by the directed transport of the axon-specific microtubuli-associated protein Tau (Ferreira et al., 1989; Caceres et al., 1991).

Whether axon outgrowth is also indicative of acquisition of cell polarity in our tectal neurons, or whether polarity is already established during the emergence of the apical primary process cannot be decided at present. In comparing the development of hippocampal and tectal neurons, one may propose that the branches at the tip of the primary process in phase 1 represent the functional equivalent of the so-called minor processes that emerge from the somata of hippocampal neurons (Dotti et al., 1988). In both types of neurons, their appearance precedes differentiation of the axon (and of the dendrites). However, the minor processes of hippocampal neurons, which do not become axonal, differentiate into dendrites, as evidenced by both morphologic and molecular criteria (Dotti et al., 1988; reviewed in Craig and Banker, 1994).

This is not observed for the exploratory branches at the tip of the primary process of tectal neurons which instead are retracted (Fig. 7). To make dendrites, tectal neurons produce new branches at the primary process. The exploratory branches of tectal neurons in phase 1 may interact with the local environment, resulting in the extension of the single efferent axon. Axon elongation may be a prerequisite for production of dendrites, as seems to be the case with hippocampal neurons (Dotti et al., 1988) and cerebellar macroneurons in culture (Caceres et al., 1991). The timely differentiation of dendrites is prevented in experiments which interfere with the
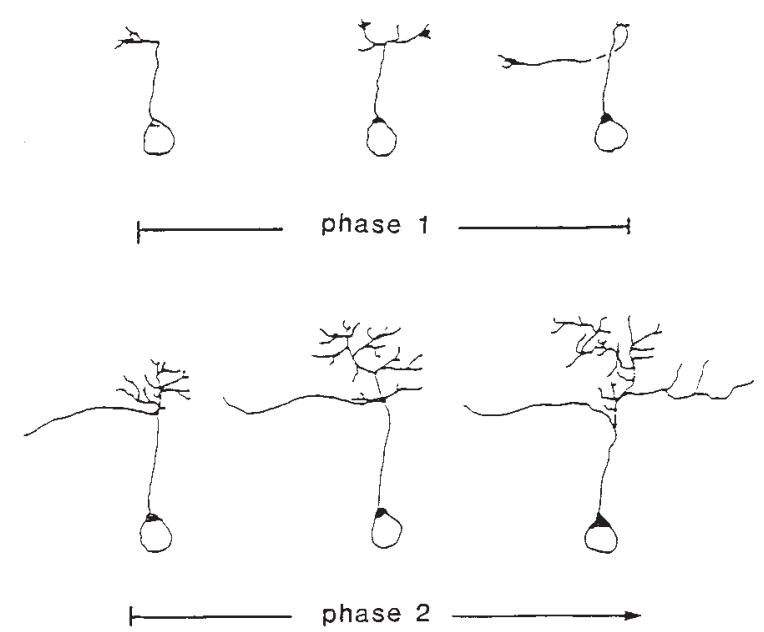

Figure 7 Summary of neuronal shape changes. This semidiagramatic drawing is composed from observations of neurons 1 and 6 in Figures 3 and 2, respectively, and neuron 8 in Figure 6, to illustrate the morphologic changes over the so-called phases 1 and 2 . The long unbranched process to the left of the neuron in phase 2 represents the axon whose path is illustrated in Figure 1. The arrowhead (phase 2 ) indicates that the morphologic changes of the dendritic tree may continue beyond $110 \mathrm{hpF}$. 
differentiation of the axon (Dotti and Banker, 1987; Caceres et al., 1991). In view of the molecular differentiation of hippocampal and other cultured neurons (reviewed in Craig and Banker, 1994) it will be interesting to explore how zebrafish tectal neurons - with the single apical process as point of origin for axons and dendrites - distribute the relevant molecules to the axon and to the dendrites.

Such an analysis may be feasible in culture. A culture of these neurons might also reveal whether their specific shape and the origin of the axon are determined intrinsically by the neuron, or whether tissue-specific cues trigger axon growth from the apical process.

The time-lapse recordings of neurons in vivo not only allow analysis of the sequence of process differentiation, they also show the impressive branching activity that accompanies axogenesis and dendritic tree formation (Berry, 1991). Also, since the neurons are in their proper and intact environment, the recordings show when and where branches are made or retracted (see below).

The technique of videorecording developing neuronal processes in vivo and the use of carbocyanine vital dyes have been discussed in detail in earlier studies which focused on retinal axons (see Methods). In addition to temporal limitations, there are spatial constraints for the recordings. One is the limited visual field of the $\times 100$ lens. The high resolution of this lens, however, was advantageous for resolving the finest branches in sufficient detail (Fig. 4). Transitional events outside the viewed segment necessarily remain unrecorded. Branches which were added and retained while observing other portions, however, were registered by focusing along the entire extent of the labeled neuron after the time-lapse recording was completed. Moreover, tracing the path of the efferent growth cone inevitably involves neglecting the activity at the primary process, and vice versa. Some fish and their labeled neurons survive for more than $24 \mathrm{~h}$ while videorecording is performed, showing that dendritic tree development lasts at least as long as the longest observation period. It almost certainly continues beyond this time, since the neurons differ in size and complexity from those of older larvae (Wilm and Fritzsch, 1989) and adults (Meek, 1983). The dendrites in adults also entertain branches in tectal layers in which retinal axons are not the predominant afferents (Davis and Northcutt, 1983; Meek, 1983). How these layers arise is beyond the scope of this study. The present results predict, however, that similar exploratory branching events are involved.
We are unable to determine whether all neurons under consideration here are representatives of one type. However, in all except one, axons departing from the dendrite were identified, implying that they belong to the class of efferent neurons. The path of axons followed here directly is indicative of neurons projecting, for instance, into the tractus tectobulbaris (Meek, 1983; Davis and Northcutt, 1983) and other targets outside the tectum. Neurons with efferent axons may be among the first of those differentiating in the embryonic zebrafish tectum to establish functional circuits. That such circuits exist is evidenced by the fact that zebrafish recognize and catch prey soon after hatching.

\section{Why Do Neurons Branch Extensively?}

The term "exploratory" branching previously used to describe the growth behavior of retinal axon arbors is applied here to tectal neurons to indicate that branch emission and retraction are evidence of the neuron's interaction with its environment. The observation that branching occurs in defined periods and zones speaks for this view. The primary process extends exploratory branches and growth cones only after it reaches the ventral aspect of the developing neuropil, suggesting that there may be stimuli [growth factors (McAllister et al, 1995), cell bound proteins, etc.] available that elicit branching. Also there should be growth promoting substrates and guidance cues near this zone to allow the directed growth of the efferent axons.

Dendritic branches could also read specific cues provided by tectal tissue (Hirata et al., 1993) for recognition of the appropriate layer. The selectivity of dendritic branches for specific layers observed in adult laminated brain structures (Berry, 1991) suggests that some sort of positional depth information is available to growing dendrites. Moreover, dendrites of related but functionally distinct neurons such as ganglion cells in the retina occupy distinct domains (Wassle et al., 1981). This speaks for a mutual recognition among dendrites of neighboring neurons. Exploratory branches may be needed for this territorial behavior. Thus, developing neurons appear to acquire their functional morphology and layer-specific branching pattern through production of excessive transitory processes (Yamaski and Ramoa, 1993) and process selection. One may envision that branches of retinal axonal arbors and those of the developing dendritic tree may touch and explore each other, since both exhibit their exploratory activity simultaneously within the tectal neuropil 
(Kaethner and Stuermer, 1992). Labeling each with different fluorescent dyes would allow observation of this proposed interaction.

The behavior of fish tectal neurons reported here may indeed be characteristic of many types of neurons in the vertebrate nervous system whose dendritic trees span several layers and receive layerspecific input from other neurons. Furthermore, a number of molecules associated with and relevant to neuronal differentiation in the zebrafish CNS, including its retinotectal system, have been identified. The cell recognition/adhesion molecule neurolin, for instance, is present on differentiating tectal neurons and their efferent axons during the time of development considered here (U. Laessing and C.A.O. Stuermer, unpublished observations). In the retina, neurolin expression is indicative of retinal ganglion cell differentiation (Laessing and Stuermer, 1996). Injection of function-blocking antibodies into zebrafish embryos disrupts the timely differentiation of distinct neuron groups (Bastmeyer et al., 1996). Thus, application of probes interfering with the function of relevant molecules can now be used to analyze the molecular determination of tectal neuron growth.

The authors thank M. A. Cahill for helping with the English text. This work was supported by the Deutsche Forschungsgemeinschaft, Grant SFB 156, to C.A.O.S.

\section{REFERENCES}

BAAs, P. W., Black, M. M., and Banker, G. A. (1989). Changes in microtubule polarity orientation during the development of hippocampal neurons in culture. $J$. Cell. Biol. 109:3085-3094.

Bastmeyer, M., Ott, H., LAessing, U., and Stuermer, C.A.O. (1996). Functional characterization of two cell adhesion molecules - the E587 antigen and neurolin during zebrafish CNS development. Soc. Neurosci. Abstr. 26:1714.

BERRY, M. (1991). Dendritic morphology and factors affecting the pattern of dendritic growth. In: Development and Plasticity of the Visual System. J. R. CronlyDillon, Ed. Macmillan, London, pp. 148-170.

Burton, P. R., and Paige, J. L. (1981). Polarity of axoplasmic microtubules in the olfactory nerve of the frog. Proc. Natl. Acad. Sci. USA 78:3269-3273.

Caceres, A., Potrebic, S. and Kosik, K. S. (1991). The effect of tau antisense oligonucleotides on neurite formation of cultured cerebellar macroneurons. J. Neurosci. 11:1515-1523.
Craig, A. M., and Banker, G. (1994). Neuronal polarity. Annu. Rev. Neurosci. 17:267-310.

CuÉnod, M., and Streit, P. (1979). Amino acid transmitters and local circuitry in optic tectum. In: The Neurosciences. F. O. Schmitt, F. G. Warden, Eds. MIT, Cambridge, MA, pp. 989-1004.

DAvis, R. E., and Northcutt, R. G. (1983). Fish Neurobiology, Vol. 2. University of Michigan Press, Ann Arbor, MI.

Deitsch, J. S., and BAnKer, G. A. (1993). An electron microscopic analysis of hippocampal neurons developing in culture: early stages in the emergence of polarity. J. Neurosci. 13:4301-4315.

Diaz, H., Lorenzo, A., Carrer, H. F., and Caceres, A. (1992). Time lapse study of neurite growth in hypothalamic dissociated neurons in culture: sex differences and estrogen effects. J. Neurosci. Res. 33:266-281.

Dotti, C. G., Sullivan, C. A., and Banker, G. A. (1988). The establishment of polarity by hippocampal neurons in culture. J. Neurosci. 8:1454-1468.

Ferreira, A., Busciglio, J., and Caceres, A. (1989). Microtubule formation and neurite growth in cerebellar macroneurons which develop in vitro: evidence for the involvement of the microtubule-associated proteins MAP-1a HMW-MAP2 and Tau. Dev. Brain Res. 49:215-228.

Goslin, K., Schreyer, D. J., Skene, P. J. H., and BANKER, G. (1988). Development of neuronal polarity: Gap-43 distinguishes axonal from dendritic growth cones. Nature 336:672-674.

Harris, W. A., Holt, C. A., and Bonhoeffer, F. (1987). Retinal axons with and without their somata, growing to and arborizing in the tectum of Xenopus embryos: a time lapse video study of single fibres in vivo. Development 101:123-133.

Heidemann, S. R., Landers, J. M., and Hamborg, M. A. (1981). Polarity orientation of axonal microtubules. $J$. Cell Biol. 91:661-665.

Hirata, T., Takagi, S., and Fujisawa, H. (1993). The membrane protein A 5, a putative neuronal recognition molecule, promotes neurite outgrowth. Neurosci. Res. 17:159-169.

Honig, M. G., and Hume, R. J. (1986). Fluorescent carboyanine dyes allow living neurons of identified origin to be studied in long term cultures. J. Cell Biol. 103: $171-187$.

Hughes, T. E. (1990). A light and electron microscopic investigation of the optic tectum of the frog Rana pipiens. II: the neurons that give rise to the crossed tectobulbar pathway. Vis. Neurosci. 4:519-531.

KAethner, R. J., and Stuermer, C. A. O. (1992). Dynamics of terminal arbor formation in living zebrafish embryos: a time lapse study of single axons. J. Neurosci. 12:3257-3271.

Kaethner, R. J., and Stuermer, C. A. O. (1994a). Growth behavior of retinotectal axons in live zebrafish 
embryos under TTX-induced neural impulse blockade. J. Neurobiol. 25:781-796.

Kaethner, R. J., and Stuermer, C. A. O. (1994b). Dynamics of dendrite development in the optic rectum of live zebrafish embryos. Soc. Neurosci. Abstr. 20:1081.

LAessing, U., and Stuermer, C. A. O. (1996). Spatiotemporal pattern of retinal ganglion cell differentiation revealed by the expression of neurolin in embryonic zebrafish. J. Neurobiol. 29:65-74.

Mc Allister, A. K., Lo, D. C., and Katz, L. C. (1995). Neurotrophins regulate dendritic growth in developing visual cortex. Neuron 15:791-803.

MeEK, J. (1983). Functional anatomy of the tectum mesencephali of the goldfish. Brain Res. Rev. 6:247-297.

Meek, J., and Schellart, N. A. M. (1978). A golgi study of goldfish optic tectum. J. Comp. Neurol. 182:89-122.

O'Rourke, N. A., Cline, H. T., and Fraser, S. E. (1994). Rapid remodeling of retinal arbors in the tectum with and without blockade of synaptic transmission. Neuron 12:921-934.

O'Rourke, N. A., and Fraser, S. E. (1990). Dynamic changes in optic fiber terminal arbors lead to retino- topic map formation: an in vivo confocal microscope study. Neuron 5:159-171.

Ramon y Cajal, S. (1911). Histologie du Système Nerveux de l'Homme et des Vertébrés, Vol 2. Paris, Maloine.

Stuermer, C. A. O. (1984). Rules of retinotectal arborizations. J. Comp. Neurol. 229:214-232.

StUERMER, C. A. O. (1988). Retinotopic organization of the developing retinotectal projection in zebrafish embryos. J. Neurosci. 8:4513-4530.

VANEGAS, H., LAUfER, M., and Amat, J. (1974). The optic tectum of a perciform teleost. I. General configuration and cytoarchitecture. J. Comp. Neurol. 154:4360.

Wässle, H., Peichl, L., and Boycott, B. B. (1981). Dendritic territories of cat retinal ganglion cells. Nature 292:344-345.

WiLM, C., and Fritzsch, B. (1989). Development of tectal neurons in the perciform teleost Haplochromis burtoni. A golgi study. Dev. Brain Res. 47:35-52.

YAMASKI, E. N., and RAMOA, A. S. (1993). Dendritic remodelling of retinal ganglion cells during development in the rat. J. Comp. Neurol. 329:277-289. 